\title{
One Diophantine inequality with integer and prime variables
}

\author{
Yongqiang Yang ${ }^{1}$ and Weiping $\mathrm{Li}^{2^{*}}$
}

"Correspondence: wpliyh@163.com ${ }^{2}$ Department of Mathematics and Information Science, Henan University of Economics and Law, Zhengzhou, 450046, P.R. China Full list of author information is available at the end of the article

\begin{abstract}
In this paper, we show that if $\lambda_{1}, \lambda_{2}, \lambda_{3}, \lambda_{4}$ are positive real numbers, at least one of the ratios $\lambda_{j} / \lambda_{j}(1 \leq i<j \leq 4)$ is irrational, then the inequality

$\left|\lambda_{1} x_{1}^{2}+\lambda_{2} x_{2}^{3}+\lambda_{3} x_{3}^{4}+\lambda_{4} x_{4}^{5}-p-\frac{1}{2}\right|<\frac{1}{2}$ has infinite solutions with natural numbers $x_{1}$, $x_{2}, x_{3}, x_{4}$ and prime $p$.
\end{abstract}

MSC: 11D75; 11P55

Keywords: Davenport-Heilbronn method; prime; Diophantine approximation

\section{Introduction}

Diophantine inequalities with integer or prime variables have been considered by many scholars. The present paper investigates one diophantine inequality with integer and prime variables. Using the Davenport-Heilbronn method, we establish our result as follows.

Theorem 1.1 Let $\lambda_{1}, \lambda_{2}, \lambda_{3}, \lambda_{4}$ be positive real numbers, at least one of the ratios $\lambda_{i} / \lambda_{j}$ $(1 \leq i<j \leq 4)$ is irrational. Then the inequality

$$
\left|\lambda_{1} x_{1}^{2}+\lambda_{2} x_{2}^{3}+\lambda_{3} x_{3}^{4}+\lambda_{4} x_{4}^{5}-p-\frac{1}{2}\right|<\frac{1}{2}
$$

has infinite solutions with natural numbers $x_{1}, x_{2}, x_{3}, x_{4}$ and prime $p$.

\section{Notation and outline of the proof}

Throughout, we use $p$ to denote a prime number and $x_{j}$ to denote a natural number. We denote by $\delta$ a sufficiently small positive number and by $\varepsilon$ an arbitrarily small positive number. Constants, both explicit and implicit, in Landau or Vinogradov symbols may depend on $\lambda_{1}, \lambda_{2}, \lambda_{3}, \lambda_{4}$. We write $e(x)=\exp (2 \pi i x)$. We use $[x]$ to denote the integer part of real variable $x$. We take $X$ to be the basic parameter, a large real integer. Since at least one of the ratios $\lambda_{i} / \lambda_{j}(1 \leq i<j \leq 4)$ is irrational, without loss of generality we may assume that $\lambda_{1} / \lambda_{2}$ is irrational. For the other cases, the only difference is in the following intermediate region, and we may deal with the same method in Section 4.

Since $\lambda_{1} / \lambda_{2}$ is irrational, then there are infinitely many pairs of integers $q$, $a$ with $\mid \lambda_{1} / \lambda_{2}-$ $a / q \mid \leq q^{-2},(a, q)=1, q>0$ and $a \neq 0$. We choose $q$ to be large in terms of $\lambda_{1}, \lambda_{2}, \lambda_{3}, \lambda_{4}$ and

(c) 2015 Yang and Li. This article is distributed under the terms of the Creative Commons Attribution 4.0 International License (http://creativecommons.org/licenses/by/4.0/), which permits unrestricted use, distribution, and reproduction in any medium, provided you give appropriate credit to the original author(s) and the source, provide a link to the Creative Commons license, and indicate if changes were made. 
make the following definitions:

$$
\begin{array}{lc}
N \asymp X^{2}, \quad L=\log N, \quad\left[N^{1-8 \delta}\right]=q, \quad \tau=N^{-1+\delta}, \\
Q=\left(\left|\lambda_{1}\right|^{-1}+\left|\lambda_{2}\right|^{-1}\right) N^{1-\delta}, \quad P=N^{6 \delta}, & T=N^{\frac{1}{3}} .
\end{array}
$$

Let $v$ be a positive real number, we define

$$
\begin{array}{ll}
K_{v}(\alpha)=v\left(\frac{\sin \pi v \alpha}{\pi v \alpha}\right)^{2}, & \alpha \neq 0, \quad K_{v}(0)=v, \\
F_{1}(\alpha)=\sum_{1 \leq x \leq X} e\left(\alpha x^{2}\right), & F_{2}(\alpha)=\sum_{1 \leq x \leq X^{\frac{2}{3}}} e\left(\alpha x^{3}\right), \quad F_{3}(\alpha)=\sum_{1 \leq x \leq X^{\frac{1}{2}}} e\left(\alpha x^{4}\right), \\
F_{4}(\alpha)=\sum_{1 \leq x \leq X^{\frac{2}{5}}} e\left(\alpha x^{5}\right), & G(\alpha)=\sum_{p \leq N}(\log p) e(\alpha p), \\
f_{1}(\alpha)=\int_{1}^{X} e\left(\alpha x^{2}\right) d x, & f_{2}(\alpha)=\int_{1}^{X^{\frac{2}{3}}} e\left(\alpha x^{3}\right) d x, \quad f_{3}(\alpha)=\int_{1}^{X^{\frac{1}{2}}} e\left(\alpha x^{4}\right) d x, \\
f_{4}(\alpha)=\int_{1}^{X^{\frac{2}{5}}} e\left(\alpha x^{5}\right) d x, & g(\alpha)=\int_{1}^{N} e(\alpha x) d x .
\end{array}
$$

It follows from (2.1) that

$$
\begin{aligned}
& K_{\nu}(\alpha) \ll \min \left(v, v^{-1}|\alpha|^{-2}\right), \\
& \int_{-\infty}^{+\infty} e(\alpha y) K_{v}(\alpha) d \alpha=\max \left(0,1-v^{-1}|y|\right) .
\end{aligned}
$$

From (2.3) it is clear that

$$
\begin{aligned}
J & =: \int_{-\infty}^{+\infty} \prod_{i=1}^{4} F_{i}\left(\lambda_{i} \alpha\right) G(-\alpha) e\left(-\frac{1}{2} \alpha\right) K_{\frac{1}{2}}(\alpha) d \alpha \\
& \leq \log \sum_{\substack{\left|\lambda_{1} x_{1}^{2}+\lambda_{2} x_{2}^{3}+\lambda_{3} x_{3}^{4}+\lambda_{4} x_{4}^{5}-p-\frac{1}{2}\right|<\frac{1}{2} \\
1 \leq x_{1} \leq X, 1 \leq x_{2} \leq X^{2 / 3}, 1 \leq x_{3} \leq X^{1 / 2}, 1 \leq x_{4} \leq X^{2 / 5}, p \leq N}} \\
& =:(\log N) \mathcal{N}(X),
\end{aligned}
$$

thus

$$
\mathcal{N}(X) \geq(\log N)^{-1} J
$$

To estimate $J$, we split the range of infinite integration into three sections, traditional named the neighborhood of the origin $\mathfrak{C}=\{\alpha \in \mathbb{R}:|\alpha| \leq \tau\}$, the intermediate region $\mathfrak{D}=$ $\{\alpha \in \mathbb{R}: \tau<|\alpha| \leq P\}$, the trivial region $\mathfrak{c}=\{\alpha \in \mathbb{R}:|\alpha|>P\}$.

To prove Theorem 1.1, we shall establish that

$$
J(\mathfrak{C}) \gg X^{\frac{77}{30}}, \quad J(\mathfrak{D})=o\left(X^{\frac{70}{33}}\right), \quad J(\mathfrak{c})=o\left(X^{\frac{77}{30}}\right)
$$


in Sections 3, 4 and 5, respectively. Thus

$$
J \gg X^{\frac{77}{30}}, \quad \mathcal{N}(X) \gg X^{\frac{77}{30}} L^{-1},
$$

and Theorem 1.1 can be established.

\section{The neighborhood of the origin}

Lemma 3.1 If $\alpha=a / q+\beta$, where $(a, q)=1$, then

$$
\sum_{1 \leq x \leq N^{1 / t}} e\left(\alpha x^{t}\right)=q^{-1} \sum_{m=1}^{q} e\left(a m^{t} / q\right) \int_{1}^{N^{1 / t}} e\left(\beta y^{t}\right) d y+O\left(q^{1 / 2+\varepsilon}(1+N|\beta|)\right) .
$$

Proof This is Theorem 4.1 of Vaughan [1].

If $|\alpha| \in \mathfrak{C}$, by Lemma 3.1, taking $a=0, q=1$, then

$$
F_{i}(\alpha)=f_{i}(\alpha)+O\left(X^{2 \delta}\right), \quad i=1,2,3,4
$$

Lemma 3.2 Let $\rho=\beta+i \gamma$ be a typical zero of the Riemann zeta function, $C$ be a positive constant,

$$
I(\alpha)=\sum_{|\gamma| \leq T, \beta \geq \frac{2}{3}} \sum_{n \leq N} n^{\rho-1} e(n \alpha), \quad J(\alpha)=O\left((1+|\alpha| N) N^{\frac{2}{3}} L^{C}\right),
$$

then

$$
\begin{aligned}
& G(\alpha)=g(\alpha)-I(\alpha)+J(\alpha), \\
& \int_{-\frac{1}{2}}^{\frac{1}{2}}|I(\alpha)|^{2} d \alpha \ll N \exp \left(-L^{\frac{1}{5}}\right), \\
& \int_{-\tau}^{\tau}|J(\alpha)|^{2} d \alpha \ll N \exp \left(-L^{\frac{1}{5}}\right) .
\end{aligned}
$$

Proof Equations (3.2), (3.3), (3.4) can be seen from Lemma 5, (29) and (33) given by Vaughan [2].

Lemma 3.3 We have

$$
\begin{aligned}
& \int_{-\frac{1}{2}}^{\frac{1}{2}}\left|f_{1}(\alpha)\right|^{2} d \alpha \ll L^{2}, \quad \int_{-\frac{1}{2}}^{\frac{1}{2}}\left|f_{2}(\alpha)\right|^{2} d \alpha \ll X^{-\frac{2}{3}} L^{2} . \\
& \int_{-\frac{1}{2}}^{\frac{1}{2}}\left|f_{3}(\alpha)\right|^{2} d \alpha \ll X^{-1} L^{2}, \quad \int_{-\frac{1}{2}}^{\frac{1}{2}}\left|f_{4}(\alpha)\right|^{2} d \alpha \ll X^{-\frac{6}{5}} L^{2} .
\end{aligned}
$$

Proof These results are from (5.16) of Vaughan [3].

Lemma 3.4 We have

$$
\int_{\mathfrak{C}}\left|\prod_{i=1}^{4} F_{i}\left(\lambda_{i} \alpha\right) G(-\alpha)-\prod_{i=1}^{4} f_{i}\left(\lambda_{i} \alpha\right) g(-\alpha)\right| K_{\frac{1}{2}}(\alpha) d \alpha \ll X^{\frac{77}{30}} L^{-1}
$$


Proof It is obvious that $F_{1}\left(\lambda_{1} \alpha\right) \ll X, f_{1}\left(\lambda_{1} \alpha\right) \ll X, F_{2}\left(\lambda_{2} \alpha\right) \ll X^{\frac{2}{3}}, f_{2}\left(\lambda_{1} \alpha\right) \ll X^{\frac{2}{3}}$, $F_{3}\left(\lambda_{3} \alpha\right) \ll X^{\frac{1}{2}}, f_{3}\left(\lambda_{3} \alpha\right) \ll X^{\frac{1}{2}}, F_{4}\left(\lambda_{4} \alpha\right) \ll X^{\frac{2}{5}}, f_{4}\left(\lambda_{4} \alpha\right) \ll X^{\frac{2}{5}}, G(-\alpha) \ll N, g(-\alpha) \ll N$,

$$
\begin{aligned}
& \prod_{i=1}^{4} F_{i}\left(\lambda_{i} \alpha\right) G(-\alpha)-\prod_{i=1}^{4} f_{i}\left(\lambda_{i} \alpha\right) g(-\alpha) \\
& =\left(F_{1}\left(\lambda_{1} \alpha\right)-f_{1}\left(\lambda_{1} \alpha\right)\right) \prod_{i=2}^{4} F_{i}\left(\lambda_{i} \alpha\right) G(-\alpha)+\left(F_{2}\left(\lambda_{2} \alpha\right)-f_{2}\left(\lambda_{2} \alpha\right)\right) \prod_{\substack{i=1 \\
i \neq 2}}^{4} F_{i}\left(\lambda_{i} \alpha\right) G(-\alpha) \\
& \quad+\left(F_{3}\left(\lambda_{3} \alpha\right)-f_{3}\left(\lambda_{3} \alpha\right)\right) \prod_{\substack{i=1 \\
i \neq 3}}^{4} F_{i}\left(\lambda_{i} \alpha\right) G(-\alpha)+\left(F_{4}\left(\lambda_{4} \alpha\right)-f_{4}\left(\lambda_{4} \alpha\right)\right) \prod_{i=1}^{3} f_{i}\left(\lambda_{i} \alpha\right) G(-\alpha) \\
& \quad+\prod_{i=1}^{4} f_{i}\left(\lambda_{i} \alpha\right)(G(-\alpha)-g(-\alpha)) .
\end{aligned}
$$

Then by (3.1), Lemmas 3.2 and 3.3,

$$
\begin{aligned}
& \int_{\mathfrak{C}}\left|\left(F_{1}\left(\lambda_{1} \alpha\right)-f_{1}\left(\lambda_{1} \alpha\right)\right) \prod_{i=2}^{4} F_{i}\left(\lambda_{i} \alpha\right) G(-\alpha)\right| K_{\frac{1}{2}}(\alpha) d \alpha \ll N^{-1+\delta} X^{2 \delta} X^{\frac{2}{3}+\frac{1}{2}+\frac{2}{5}} N \ll X^{\frac{47}{30}+4 \delta}, \\
& \int_{\mathfrak{C}}\left|\prod_{i=1}^{4} f_{i}\left(\lambda_{i} \alpha\right)(G(-\alpha)-g(-\alpha))\right| K_{\frac{1}{2}}(\alpha) d \alpha \\
& \quad \ll X^{\frac{47}{30}}\left(\int_{\mathfrak{C}}\left|f_{1}\left(\lambda_{1} \alpha\right)\right|^{2} K_{\frac{1}{2}}(\alpha) d \alpha\right)^{\frac{1}{2}}\left(\int_{\mathfrak{C}}|J(-\alpha)-I(-\alpha)|^{2} K_{\frac{1}{2}}(\alpha) d \alpha\right)^{\frac{1}{2}} \\
& \quad \ll X^{\frac{47}{30}}\left(\int_{-\frac{1}{2}}^{\frac{1}{2}}\left|f_{1}\left(\lambda_{1} \alpha\right)\right|^{2} d \alpha\right)^{\frac{1}{2}}\left(\int_{\mathfrak{C}}|J(\alpha)|^{2} d \alpha+\int_{-\frac{1}{2}}^{\frac{1}{2}}|I(\alpha)|^{2} d \alpha\right)^{\frac{1}{2}} \\
& \ll X^{\frac{47}{30}} L\left(N \exp \left(-L^{\frac{1}{5}}\right)\right)^{\frac{1}{2}} \\
& \ll X^{\frac{77}{30}} L^{-1} .
\end{aligned}
$$

The other cases are similar, and the proof of Lemma 3.4 is completed.

\section{Lemma 3.5 We have}

$$
\int_{|\alpha|>N^{-1+\delta}}\left|\prod_{i=1}^{4} f_{i}\left(\lambda_{i} \alpha\right) g(-\alpha)\right| K_{\frac{1}{2}}(\alpha) d \alpha \ll X^{\frac{77}{30}-\frac{77}{30} \delta} .
$$

Proof It follows from Vaughan [1] that for $\alpha \neq 0$,

$$
\begin{array}{ll}
f_{1}\left(\lambda_{1} \alpha\right) \ll|\alpha|^{-\frac{1}{2}}, & f_{2}\left(\lambda_{2} \alpha\right) \ll|\alpha|^{-\frac{1}{3}}, \quad f_{3}\left(\lambda_{3} \alpha\right) \ll|\alpha|^{-\frac{1}{4}}, \\
f_{4}\left(\lambda_{4} \alpha\right) \ll|\alpha|^{-\frac{1}{5}}, & g(-\alpha) \ll|\alpha|^{-1} .
\end{array}
$$

Thus

$$
\int_{|\alpha|>N^{-1+\delta}}\left|\prod_{i=1}^{4} f_{i}\left(\lambda_{i} \alpha\right) g(-\alpha)\right| K_{\frac{1}{2}}(\alpha) d \alpha \ll \int_{|\alpha|>N^{-1+\delta}}|\alpha|^{-\frac{137}{60}} d \alpha \ll X^{\frac{77}{30}-\frac{77}{30} \delta} .
$$


Lemma 3.6 We have

$$
\int_{-\infty}^{+\infty} \prod_{i=1}^{4} f_{i}\left(\lambda_{i} \alpha\right) g(-\alpha) e\left(-\frac{1}{2} \alpha\right) K_{\frac{1}{2}}(\alpha) d \alpha \gg X^{\frac{77}{30}}
$$

Proof From (2.3), one has

$$
\begin{aligned}
\int_{-\infty}^{+\infty} & \prod_{i=1}^{4} f_{i}\left(\lambda_{i} \alpha\right) g(-\alpha) e\left(-\frac{1}{2} \alpha\right) K_{\frac{1}{2}}(\alpha) d \alpha \\
= & \int_{1}^{X} \int_{1}^{X^{\frac{2}{3}}} \int_{1}^{X^{\frac{1}{2}}} \int_{1}^{X^{\frac{2}{5}}} \int_{1}^{N} \int_{-\infty}^{+\infty} e\left(\alpha\left(\sum_{i=1}^{4} \lambda_{i} x_{i}^{1+i}-x-\frac{1}{2}\right)\right) \\
& \cdot K_{\frac{1}{2}}(\alpha) d \alpha d x d x_{4} \cdots d x_{1} \\
= & \frac{1}{120} \int_{1}^{X^{2}} \cdots \int_{1}^{X^{2}} \int_{1}^{N} \int_{-\infty}^{+\infty} x_{1}^{-\frac{1}{2}} x_{2}^{-\frac{2}{3}} x_{3}^{-\frac{3}{4}} x_{4}^{-\frac{4}{5}} e\left(\alpha\left(\sum_{i=1}^{4} \lambda_{i} x_{i}-x-\frac{1}{2}\right)\right) \\
& \cdot K_{\frac{1}{2}}(\alpha) d \alpha d x d x_{4} \cdots d x_{1} \\
= & \frac{1}{120} \int_{1}^{X^{2}} \cdots \int_{1}^{X^{2}} \int_{1}^{N} x_{1}^{-\frac{1}{2}} x_{2}^{-\frac{2}{3}} x_{3}^{-\frac{3}{4}} x_{4}^{-\frac{4}{5}} \\
& \cdot \max \left(0, \frac{1}{2}-\left|\sum_{i=1}^{4} \lambda_{i} x_{i}-x-\frac{1}{2}\right|\right) d x d x_{4} \cdots d x_{1} .
\end{aligned}
$$

Let $\left|\sum_{i=1}^{4} \lambda_{i} x_{i}-x-\frac{1}{2}\right| \leq \frac{1}{4}$, then $\sum_{i=1}^{4} \lambda_{i} x_{i}-\frac{3}{4} \leq x \leq \sum_{i=1}^{4} \lambda_{i} x_{i}-\frac{1}{4}$. Based on $\sum_{i=1}^{4} \lambda_{i} x_{i}-\frac{3}{4}>$ $1, \sum_{i=1}^{4} \lambda_{i} x_{i}-\frac{1}{4}<N$, one may take

$$
\lambda_{j} X^{2}\left(8 \sum_{i=1}^{4} \lambda_{i}\right)^{-1} \leq x_{j} \leq \lambda_{j} X^{2}\left(4 \sum_{i=1}^{4} \lambda_{i}\right)^{-1}, \quad j=1, \ldots, 4,
$$

hence

$$
\int_{-\infty}^{+\infty} \prod_{i=1}^{4} f_{i}\left(\lambda_{i} \alpha\right) g(-\alpha) e\left(-\frac{1}{2} \alpha\right) K_{\frac{1}{2}}(\alpha) d \alpha \geq \frac{1}{960} \prod_{j=1}^{4} \lambda_{j}\left(8 \sum_{i=1}^{4} \lambda_{i}\right)^{-4} X^{\frac{77}{30}}
$$

This completes the proof of Lemma 3.6.

\section{The intermediate region}

Lemma 4.1 We have

$$
\begin{aligned}
& \int_{-\infty}^{+\infty}\left|F_{1}\left(\lambda_{1} \alpha\right)\right|^{4} K_{\frac{1}{2}}(\alpha) d \alpha \ll X^{2+\varepsilon}, \\
& \int_{-\infty}^{+\infty}\left|F_{2}\left(\lambda_{2} \alpha\right)\right|^{8} K_{\frac{1}{2}}(\alpha) d \alpha \ll X^{\frac{10}{3}+\frac{2}{3} \varepsilon}, \\
& \int_{-\infty}^{+\infty}\left|F_{3}\left(\lambda_{3} \alpha\right)\right|^{16} K_{\frac{1}{2}}(\alpha) d \alpha \ll X^{6+\frac{1}{2} \varepsilon}, \\
& \int_{-\infty}^{+\infty}\left|F_{4}\left(\lambda_{4} \alpha\right)\right|^{32} K_{\frac{1}{2}}(\alpha) d \alpha \ll X^{\frac{54}{5}+\frac{2}{5} \varepsilon},
\end{aligned}
$$




$$
\int_{-\infty}^{+\infty}|G(-\alpha)|^{2} K_{\frac{1}{2}}(\alpha) d \alpha \ll N L
$$

Proof By (2.2) and Hua's inequality, we have

$$
\begin{aligned}
& \int_{-\infty}^{+\infty}\left|F_{1}\left(\lambda_{1} \alpha\right)\right|^{4} K_{\frac{1}{2}}(\alpha) d \alpha \\
& \quad \ll \sum_{m=-\infty}^{+\infty} \int_{m}^{m+1}\left|F_{1}\left(\lambda_{1} \alpha\right)\right|^{4} K_{\frac{1}{2}}(\alpha) d \alpha \\
& \quad \ll \sum_{m=0}^{1} \int_{m}^{m+1}\left|F_{1}\left(\lambda_{1} \alpha\right)\right|^{4} d \alpha+\sum_{m=2}^{+\infty} m^{-2} \int_{m}^{m+1}\left|F_{1}\left(\lambda_{1} \alpha\right)\right|^{4} d \alpha \\
& \ll X^{2+\varepsilon}+X^{2+\varepsilon} \sum_{m=2}^{+\infty} m^{-2} \\
& \ll X^{2+\varepsilon} .
\end{aligned}
$$

The proofs of (4.2)-(4.5) are similar to (4.1).

\section{Lemma 4.2 We have}

$$
\int_{-\infty}^{+\infty}\left|F_{1}\left(\lambda_{1} \alpha\right)\right|^{2}\left|F_{3}\left(\lambda_{3} \alpha\right)\right|^{4} K_{\frac{1}{2}}(\alpha) d \alpha \ll X^{2+\varepsilon}
$$

Proof Firstly, we consider the number of solutions $R(X, Z)$ of equation

$$
\lambda_{1}\left(x_{1}^{2}-x_{2}^{2}\right)=\lambda_{j}\left(y_{1}^{4}+y_{2}^{4}-y_{3}^{4}-y_{4}^{4}\right), \quad 1 \leq x_{1}, x_{2} \leq X, 1 \leq y_{1}, y_{2}, y_{3}, y_{4} \leq Z
$$

If $x_{1}=x_{2}$, then $R(X, Z) \ll X^{\varepsilon} X Z^{2}$, and if $x_{1} \neq x_{2}$, then $R(X, Z) \ll X^{\varepsilon} Z^{4}$. We take $Z=X^{\frac{1}{2}}$, then $R(X, Z) \ll X^{2+\varepsilon}$.

$$
\begin{aligned}
& \int_{-\infty}^{+\infty}\left|F_{1}\left(\lambda_{1} \alpha\right)\right|^{2}\left|F_{3}\left(\lambda_{3} \alpha\right)\right|^{4} K_{\frac{1}{2}}(\alpha) d \alpha \\
& \quad \ll \sum_{m=-\infty}^{+\infty} \int_{m}^{m+1}\left|F_{1}\left(\lambda_{1} \alpha\right)\right|^{2}\left|F_{3}\left(\lambda_{3} \alpha\right)\right|^{4} K_{\frac{1}{2}}(\alpha) d \alpha \\
& \quad \ll \sum_{m=0}^{1} \int_{m}^{m+1}\left|F_{1}\left(\lambda_{1} \alpha\right)\right|^{2}\left|F_{3}\left(\lambda_{3} \alpha\right)\right|^{4} d \alpha+\sum_{m=2}^{+\infty} m^{-2} \int_{m}^{m+1}\left|F_{1}\left(\lambda_{1} \alpha\right)\right|^{2}\left|F_{3}\left(\lambda_{3} \alpha\right)\right|^{4} d \alpha \\
& \ll X^{2+\varepsilon} .
\end{aligned}
$$

Lemma 4.3 Suppose that $(a, q)=1,|\alpha-a / q| \leq q^{-2}, \phi(x)=\alpha x^{k}+\alpha_{1} x^{k-1}+\cdots+\alpha_{k-1} x+\alpha_{k}$, then

$$
\sum_{x=1}^{M} e(\phi(x)) \ll M^{1+\varepsilon}\left(q^{-1}+M^{-1}+q M^{-k}\right)^{2^{1-k}}
$$

Proof This is Lemma 2.4 (Weyl's inequality) of Vaughan [1]. 
Lemma 4.4 For every real number $\alpha \in \mathfrak{D}$, let $W(\alpha)=\min \left(\left|F_{1}\left(\lambda_{1} \alpha\right)\right|^{\frac{2}{3}},\left|F_{2}\left(\lambda_{2} \alpha\right)\right|\right)$, then

$$
W(\alpha) \ll X^{\frac{2}{3}-\frac{1}{3} \delta+\varepsilon}
$$

Proof For $\alpha \in \mathfrak{D}$ and $j=1,2$, we choose $a_{j}, q_{j}$ such that

$$
\left|\lambda_{j} \alpha-a_{j} / q_{j}\right| \leq q_{j}^{-1} Q^{-1}
$$

with $\left(a_{j}, q_{j}\right)=1$ and $1 \leq q_{j} \leq Q$.

Firstly, we note that $a_{1} a_{2} \neq 0$. Secondly, if $q_{1}, q_{2} \leq P$, then

$$
\left|a_{2} q_{1} \frac{\lambda_{1}}{\lambda_{2}}-a_{1} q_{2}\right| \leq\left|\frac{a_{2} / q_{2}}{\lambda_{2} \alpha} q_{1} q_{2}\left(\lambda_{1} \alpha-\frac{a_{1}}{q_{1}}\right)\right|+\left|\frac{a_{1} / q_{1}}{\lambda_{2} \alpha} q_{1} q_{2}\left(\lambda_{2} \alpha-\frac{a_{2}}{q_{2}}\right)\right| \ll P Q^{-1}<\frac{1}{2 q} .
$$

We recall that $q$ was chosen as the denominator of a convergent to the continued fraction for $\lambda_{1} / \lambda_{2}$. Thus, by Legendre's law of best approximation, we have $\left|q^{\prime} \frac{\lambda_{1}}{\lambda_{2}}-a^{\prime}\right|>\frac{1}{2 q}$ for all integers $a^{\prime}, q^{\prime}$ with $1 \leq q^{\prime}<q$, thus $\left|a_{2} q_{1}\right| \geq q=\left[N^{1-8 \delta}\right]$. However, from (4.6) we have $\left|a_{2} q_{1}\right| \ll q_{1} q_{2} P \ll N^{18 \delta}$, this is a contradiction. We have thus established that for at least one $j, P<q_{j} \ll Q$. Hence, Lemma 4.3 gives the desired inequality for $W(\alpha)$.

\section{Lemma 4.5 We have}

$$
\int_{\mathcal{D}} \prod_{i=1}^{4} F_{i}\left(\lambda_{i} \alpha\right) G(-\alpha) e\left(-\frac{1}{2} \alpha\right) K_{\frac{1}{2}}(\alpha) d \alpha \ll X^{\frac{77}{30}-\frac{1}{12} \delta+\varepsilon} .
$$

Proof By Lemmas 4.1, 4.2, 4.4 and Hölder's inequality, we have

$$
\begin{aligned}
\int_{\mathcal{D}} & \prod_{i=1}^{4}\left|F_{i}\left(\lambda_{i} \alpha\right) G(-\alpha)\right| K_{\frac{1}{2}}(\alpha) d \alpha \\
\ll & \max _{\alpha \in \mathcal{D}}|W(\alpha)|^{\frac{3}{16}} \int_{\mathfrak{D}}\left|F_{1}\left(\lambda_{1} \alpha\right)\right|^{\frac{7}{8}} \prod_{i=2}^{4}\left|F_{i}\left(\lambda_{i} \alpha\right) G(-\alpha)\right| K_{\frac{1}{2}}(\alpha) d \alpha \\
& +\max _{\alpha \in \mathcal{D}}|W(\alpha)|^{\frac{1}{4}} \int_{\mathcal{D}}\left|F_{2}\left(\lambda_{2} \alpha\right)\right|^{\frac{3}{4}} \prod_{\substack{i=1 \\
i \neq 2}}^{4}\left|F_{i}\left(\lambda_{i} \alpha\right) G(-\alpha)\right| K_{\frac{1}{2}}(\alpha) d \alpha \\
\ll & \left(X^{\frac{2}{3}-\frac{1}{3} \delta+\varepsilon}\right)^{\frac{3}{16}}\left(\int_{-\infty}^{+\infty}\left|F_{1}\left(\lambda_{1} \alpha\right)\right|^{4} K_{\frac{1}{2}}(\alpha) d \alpha\right)^{\frac{3}{32}}\left(\int_{-\infty}^{+\infty}\left|F_{2}\left(\lambda_{2} \alpha\right)\right|^{8} K_{\frac{1}{2}}(\alpha) d \alpha\right)^{\frac{1}{8}} \\
& \cdot\left(\int_{-\infty}^{+\infty}\left|F_{1}\left(\lambda_{1} \alpha\right)\right|^{2}\left|F_{3}\left(\lambda_{3} \alpha\right)\right|^{4} K_{\frac{1}{2}}(\alpha) d \alpha\right)^{\frac{1}{4}}\left(\int_{-\infty}^{+\infty}\left|F_{4}\left(\lambda_{4} \alpha\right)\right|^{32} K_{\frac{1}{2}}(\alpha) d \alpha\right)^{\frac{1}{32}} \\
& \cdot\left(\int_{-\infty}^{+\infty}|G(-\alpha)|^{2} K_{\frac{1}{2}}(\alpha) d \alpha\right)^{\frac{1}{2}} \\
& +\left(X^{\frac{2}{3}-\frac{1}{3} \delta+\varepsilon}\right)^{\frac{1}{4}}\left(\int_{-\infty}^{+\infty}\left|F_{1}\left(\lambda_{1} \alpha\right)\right|^{4} K_{\frac{1}{2}}(\alpha) d \alpha\right)^{\frac{1}{8}}\left(\int_{-\infty}^{+\infty}\left|F_{2}\left(\lambda_{2} \alpha\right)\right|^{8} K_{\frac{1}{2}}(\alpha) d \alpha\right)^{\frac{3}{32}} \\
& \cdot\left(\int_{-\infty}^{+\infty}\left|F_{1}\left(\lambda_{1} \alpha\right)\right|^{2}\left|F_{3}\left(\lambda_{3} \alpha\right)\right|^{4} K_{\frac{1}{2}}(\alpha) d \alpha\right)^{\frac{1}{4}}\left(\int_{-\infty}^{+\infty}\left|F_{4}\left(\lambda_{i} \alpha\right)\right|^{32} K_{\frac{1}{2}}(\alpha) d \alpha\right)^{\frac{1}{32}}
\end{aligned}
$$




$$
\begin{aligned}
& \left(\int_{-\infty}^{+\infty}|G(-\alpha)|^{2} K_{\frac{1}{2}}(\alpha) d \alpha\right)^{\frac{1}{2}} \\
\ll & \left(X^{\frac{2}{3}-\frac{1}{3} \delta+\varepsilon}\right)^{\frac{3}{16}}\left(X^{2+\varepsilon}\right)^{\frac{3}{32}}\left(X^{\frac{10}{3}+\frac{2}{3} \varepsilon}\right)^{\frac{1}{8}}\left(X^{2+\varepsilon}\right)^{\frac{1}{4}}\left(X^{\frac{54}{5}+\frac{2}{5} \varepsilon}\right)^{\frac{1}{32}}(N L)^{\frac{1}{2}} \\
& +\left(X^{\frac{2}{3}-\frac{1}{3} \delta+\varepsilon}\right)^{\frac{1}{4}}\left(X^{2+\varepsilon}\right)^{\frac{1}{8}}\left(X^{\frac{10}{3}+\frac{2}{3} \varepsilon}\right)^{\frac{3}{32}}\left(X^{2+\varepsilon}\right)^{\frac{1}{4}}\left(X^{\frac{54}{5}+\frac{2}{5} \varepsilon}\right)^{\frac{1}{32}}(N L)^{\frac{1}{2}} \\
\ll & X^{\frac{77}{30}-\frac{1}{12} \delta+\varepsilon} .
\end{aligned}
$$

\section{The trivial region}

Lemma 5.1 (Lemma 2 of [4]) Let $V(\alpha)=\sum e\left(\alpha f\left(x_{1}, \ldots, x_{m}\right)\right.$ ), where $f$ is any real function and the summation is over any finite set of values of $x_{1}, \ldots, x_{m}$. Then, for any $A>4$, we have

$$
\int_{|\alpha|>A}|V(\alpha)|^{2} K_{v}(\alpha) d \alpha \leq \frac{16}{A} \int_{-\infty}^{\infty}|V(\alpha)|^{2} K_{v}(\alpha) d \alpha
$$

Lemma 5.2 We have

$$
\int_{\mathfrak{c}} \prod_{i=1}^{4} F_{i}\left(\lambda_{i} \alpha\right) G(-\alpha) e\left(-\frac{1}{2} \alpha\right) K_{\frac{1}{2}}(\alpha) d \alpha \ll X^{\frac{77}{30}-12 \delta+\varepsilon} .
$$

Proof By Lemmas 5.1, 4.1, 4.2 and Schwarz's inequality, we have

$$
\begin{aligned}
& \int_{\mathfrak{c}} \prod_{i=1}^{4} F_{i}\left(\lambda_{i} \alpha\right) G(-\alpha) e\left(-\frac{1}{2} \alpha\right) K_{\frac{1}{2}}(\alpha) d \alpha \\
& \ll \int_{\mathfrak{c}}\left|\prod_{i=1}^{4} F_{i}\left(\lambda_{i} \alpha\right) G(-\alpha)\right| K_{\frac{1}{2}}(\alpha) d \alpha \\
& \ll \frac{1}{P} \int_{-\infty}^{+\infty}\left|\prod_{i=1}^{4} F_{i}\left(\lambda_{i} \alpha\right) G(-\alpha)\right| K_{\frac{1}{2}}(\alpha) d \alpha \\
& \ll N^{-6 \delta} \max \left|F_{4}\left(\lambda_{4} \alpha\right)\right|\left(\int_{-\infty}^{+\infty}\left|F_{1}\left(\lambda_{1} \alpha\right)\right|^{4} K_{\frac{1}{2}}(\alpha) d \alpha\right)^{\frac{1}{8}} \\
& \cdot\left(\int_{-\infty}^{+\infty}\left|F_{1}\left(\lambda_{1} \alpha\right)\right|^{2}\left|F_{3}\left(\lambda_{3} \alpha\right)\right|^{4} K_{\frac{1}{2}}(\alpha) d \alpha\right)^{\frac{1}{4}}\left(\int_{-\infty}^{+\infty}\left|F_{2}\left(\lambda_{2} \alpha\right)\right|^{8} K_{\frac{1}{2}}(\alpha) d \alpha\right)^{\frac{1}{8}} \\
& \cdot\left(\int_{-\infty}^{+\infty}|G(-\alpha)|^{2} K_{\frac{1}{2}}(\alpha) d \alpha\right)^{\frac{1}{2}} \\
& \ll N^{-6 \delta} X^{\frac{2}{5}}\left(X^{2+\varepsilon}\right)^{\frac{1}{8}+\frac{1}{4}}\left(X^{\frac{10}{3}+\frac{2}{3} \varepsilon}\right)^{\frac{1}{8}}(N L)^{\frac{1}{2}} \\
& \ll X^{\frac{77}{30}-12 \delta+\varepsilon} .
\end{aligned}
$$

\section{Competing interests}

The authors declare that they have no competing interests.

\section{Authors' contributions}

All authors contributed equally to the writing of this paper. All authors read and approved the final manuscript.

\section{Author details}

${ }^{1}$ College of Computer and Information Engineering, Henan University of Economics and Law, Zhengzhou, 450046,

P.R. China. ${ }^{2}$ Department of Mathematics and Information Science, Henan University of Economics and Law, Zhengzhou, 450046, P.R. China. 


\section{Acknowledgements}

This work is supported by the National Natural Science Foundation of China (Grant Nos. 11371122, 11471112), the Innovation Scientists and Technicians Troop Construction Projects of Henan Province (China).

Received: 26 June 2015 Accepted: 9 September 2015 Published online: 18 September 2015

\section{References}

1. Vaughan, RC: The Hardy-Littlewood Method, 2nd edn. Cambridge Tracts in Mathematics, vol. 125. Cambridge University Press, Cambridge (1997)

2. Vaughan, RC: Diophantine approximation by prime numbers, I. Proc. Lond. Math. Soc. 28, 373-384 (1974)

3. Vaughan, RC: Diophantine approximation by prime numbers, II. Proc. Lond. Math. Soc. 28, 385-401 (1974)

4. Davenport, H, Roth, KF: The solubility of certain Diophantine inequalities. Mathematika 2, 81-96 (1955)

Submit your manuscript to a SpringerOpen ${ }^{\odot}$ journal and benefit from:

- Convenient online submission

- Rigorous peer review

- Immediate publication on acceptance

- Open access: articles freely available online

- High visibility within the field

- Retaining the copyright to your article 